\title{
Asymptomatic ectopic pancreas with low grade dysplasia incidentally foundarising from the jejunum during laparoscopic mini gastric bypass: case report
}

\begin{abstract}
Introduction: Aberrant pancreas, also called heterotopic or ectopic pancreas, is defined as pancreatic tissue found outside its usual anatomic location. It is diagnosed often incidentally, and can be found at different sites in the gastrointestinal tract. Ectopic pancreas may become clinically evident when complications occur due to some pathologic changes such as inflammation, bleeding, obstruction, or malignant transformation, or may remain completely asymptomatic.
\end{abstract}

Case presentation: We present a case of a 55 years old man, morbidly obese, presenting for laparoscopic gastric mini-bypass. Incidentally, a mass was identified at the proximal jejunum. After wedge resection was done, histologic exam revealed the diagnosis of a fully developed ectopic pancreatic tissue (type I), with low grade dysplasia

Conclusion: Although ectopic pancreas is a rare diagnosis, it should be considered in the differential diagnosis of any submucosal bowel lesions. This is the first reported case of low grade dysplasia originating from a completely asymptomatic jejunal aberrant pancreatic tissue.

Keywords: ectopic pancreas, aberrant pancreas, jejunum, low grade dysplasia
Volume 8 Issue 3 - 2018

\author{
Hussein Mcheimeche, Louai S Chalaby, Riad \\ Saheli, Dany Lichaa, Chadi Nassar, Hussein \\ Moussawi, Houssam El-Dine Bitar, Samer H \\ Dbouk \\ Department of general surgery,Al Zahraa University Hospital, \\ Lebanon
}

Correspondence: Hussein Mcheimeche, Department of General Surgery, Al Zahraa University Hospital, Jnah, Beirut. Lebanon, Tel 0096|332937।, Fax 0096 I I843758, Email hmcheimeche@hotmail.com

Received: May 07, 2018| Published: May 30, 2018

\section{Introduction}

An "aberrant pancreas" is a rare congenital anomaly, described for the first time by Schultz in 1729 , but no been histologically confirmed until 1859 , by Klob. ${ }^{1}$ The ectopic pancreatic tissue is located outside the anatomic location of the pancreas, without any vascular or ductal continuity with the main gland, and it may be found in multiple areas in the abdominal cavity, mainly along the proximal gastro-intestinal tract, or within the spleen, liver and even in the biliary ducts and gallbladder. $^{2}$

From an embryological point of view, aberrant pancreas is thought to occur according to different theories. One theory states that the aberrant pancreatic tissue may be formed following the migration and settlement of a pancreatic rest outside the anatomic pancreatic location. Another theory explains that it could be due to anomalous differentiation of some pluripotent endodermal cells located within the primitive gut. ${ }^{1}$ Histologically, aberrant pancreas may contain some or all the components of the normal pancreatic, like acini, islets and ducts.

This ectopic tissue may, rarely, show any manifestation that entopic pancreas can present, such as malignant transformation or inflammation and necrosis (Acute heterotopic pancreatitis), and patients may present complaining of epigastric pain, nausea, vomiting and melena in some cases where the ectopic pancreas develops bleeding. Also, it may remain completely asymptomatic, especially where it of a small size, or it lacks mucosal involvement. ${ }^{3}$ Particularly, aberrant pancreas of the jejunum rarely develop complications, and its diagnosis is mostly incidental, during laparotomies for other purposes, or during autopsies, making the estimation of its true incidence very difficult to be determined. ${ }^{4}$

In this article, we present an extremely rare case, where a patient was found incidentally during an elective surgical procedure to have jejunal aberrant pancreas, with low grade dysplasia. This presentation of ectopic pancreas is very unusual, and seems to be the first reported case in the literature, aside from the few reported cases of symptomatic adenocarcinoma or pancreatic intra-epithelial neoplasia.

\section{Case presentation}

A 55 year old male patient, morbidly obese, with a BMI of 42.5 $\mathrm{kg} / \mathrm{m}^{2}$, who is known to have type II Diabetes Mellitus (on oral hypoglycemic) and Coronary arterial disease (s/p PTCA 10 months ago), was planned to undergo a gastric mini-bypass for management of his morbid obesity, after failure of several attempts to lose weight. Incidentally, he was found to have a jejunal mass, of yellow color, with firm and heterogeneous consistency, measuring $2 \times 2 \mathrm{~cm}$. This lesion was located only $15 \mathrm{~cm}$ distally to the ligament of treitz. It was covering less than 180 degrees of the jejunal circumference. The decision was taken to undergo a wedge resection of the mass, using a GIA stapler, which was placed in a transverse direction, to avoid the risk of small bowel lumen stenosis. The lesion was sent to pathologic exam, and the planned procedure was continued. The patient was kept NPO for 3 days, and has undergone an upper GI series on the third post-op, that showed no evidence of contrast leakage through the anastomotic or resection sites, with good transit throughout the small bowel loops. So, he was started on clear fluid and discharged home. On follow up visit, he was found to be tolerating diet well, 
with no complaints. Histopathology report revealed the presence of a fully differentiated ectopic pancreas (type I, according to Heinrich classification), with low grade dysplasia, extending in the submucosa, muscularis propria and serosa (Figure 1).

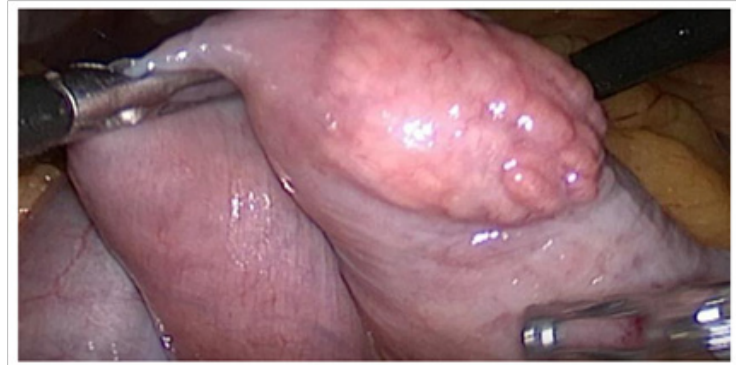

Figure 1 Incidentally discovered jejunal lesion during laparoscopic mini GBP.

\section{Discussion}

Heterotopic pancreas is defined as pancreatic tissue located separately from the primary gland, without any vascular or ductal continuity with it. It can be found in different sites within the abdominal cavity, mainly in the proximal gastro-intestinal tract (stomach and duodenum). The diagnosis is usually incidental, since most of aberrant pancreas cases are asymptomatic, and especially if it was found to be arising from the jejunum. ${ }^{4}$ Heinrich classification and its subsequent Gasper-Fuentes modification, classifies pancreatic aberrant tissue into 4 types, according to their differentiation and the presence of acini, ducts, or islets. Type I (typical pancreatic tissue) contains all three type of cells: duct, acini, and islets. Type II contains only pancreatic ducts. Type III is exocrine variety, which contains only acinar tissue. Type IV, is defined as endocrine variety, where only islet cell are identified. ${ }^{5}$ In our case, histopathologic exam findings showed evidence of well-developed acini, ducts, and islets, and was therefore considered to be type I. ${ }^{5}$

Heterotopic pancreas may remain clinically silent, but also may exhibit different changes in rare cases, leading to a wide range of complications, and becomes symptomatic. Some cases develop acute inflammation, leading to the same clinical picture of a pancreatitis with abdominal pain. ${ }^{1}$ Others may develop hemorrhage, with hematemesis. In cases where the ectopic pancreas arises within the esophagus, dysphagia may occur. Also, malignant degeneration of aberrant pancreas was reported in several cases, ranging from intra-epithelial neoplasm and carcinoma in situ, to localized adenocarcinoma, or even metastatic disease. ${ }^{6}$

Our case is one of the rare cases, where pancreatic aberrant tissue was arising from the jejunum. Similarly to the literature, where it is noted that jejunal aberrant pancreas seldom develop symptoms, our patient was completely asymptomatic, and he was incidentally found to have such anomaly. But surprisingly, the pathology revealed the evidence low grade dysplasia, which is a precursor of malignancy. Since screening tests are not available for tumors of the small bowels, such as endoscopy, most of the patients present when their tumor becomes symptomatic, and only when the tumor is already in advance stages. Our patient seems to be lucky and took advantage of the meticulous inspection of the abdominal cavity, which is an important step that is not be omitted during any laparotomy or laparoscopy. Finally, the decision to proceed with a wedge resection was made since we have found that the mass could be resected completely without any risk of stenosis of the small bowel lumen, especially with the GIA stapler oriented transversally. After wedge resection was done, there we could complete the procedure as planned preoperatively, without any contraindication or difficulty.

\section{Conclusion}

In summary, we reported a rare case of a completely asymptomatic aberrant pancreas, arising from the jejunum, which was discovered incidentally during a bariatric procedure, and turned to be positive for low grade dysplasia. This seems to be first reported case of such presentation for aberrant pancreas

\section{Consent}

Written informed consent was obtained from the patient for publication of this case report and any accompanying images.

\section{Acknowledgments}

None.

\section{Authors contributions}

$\mathrm{HM}$ and $\mathrm{LC}$ drafted the manuscript and provided the original pictures. SD, RS, DL, HB, HMo and $\mathrm{ChN}$ collected the clinical and pathological data and helped to draft the manuscript. LC and SD reviewed the manuscript. All authors read and approved the final manuscript.

\section{Conflicts of interests}

The authors declare that they have no competing interests.

\section{References}

1. González Callejas C, González Crespo F, Zurita Saavedra M, et al. Ectopic pancreas in the gallbladder. Cir Esp. 2013;91(2):130-131.

2. Yamaoka Y, Yamaguchi T, Kinugasa Y, et al. Adenocarcinoma arising from jejunal ectopic pancreas mimicking peritoneal metastasis from colon cancer: a case report and literature review. Surg Case Rep. 2015;1:114.

3. Hamabe A, Ueshima S, Akamatsu H, et al. Infected pancreatic necrosis occurring in a jejunal aberrant pancreas: report of a case. Surg Today. 2012;42:583-588.

4. Kenji Fujiwara, Chihiro Nakahara, Toru Yamasaki, et al. Adenocarcinoma of Aberrant Pancreas in the Jejunum: Report of a Case. $J$ Gastrointest Canc. 2010;41:135-137.

5. Marius Distler. Pancreatic heterotopia of the duodenum: Anatomic Anomaly or clinical challenge? J gastrointest Surg. 2011;15:631-633.

6. Saad RS, Essig DL, Silverman JF, et al. Diagnostic utility of CDX-2 expression in separating metastatic gastrointestinal adenocarcinoma from other metastatic adenocarcinoma in fine-needle aspiration cytology using cell blocks. Cancer. 2004;102:168-173. 